\title{
Effects of Visual Prompt on Recycling Behavior of Waste Batteries
}

\author{
Yuanqing Deng, Fuzhen Zhang* \\ Department of Marxism, China Pharmaceutical University, Nanjing, China \\ Email: quyq1996@163.com, *fzzhang@cpu.edu.cn
}

How to cite this paper: Deng, Y. Q., \& Zhang, F. Z. (2019). Effects of Visual Prompt on Recycling Behavior of Waste Batteries. Psychology, 10, 1565-1571.

https://doi.org/10.4236/psych.2019.1012102

Received: July 15, 2019

Accepted: September 3, 2019

Published: September 6, 2019

Copyright $\odot 2019$ by author(s) and Scientific Research Publishing Inc. This work is licensed under the Creative Commons Attribution International License (CC BY 4.0).

http://creativecommons.org/licenses/by/4.0/

\begin{abstract}
Visual prompts are a kind of visual stimulation, through vectors such as text, pictures, bar charts, and image data which guide and persuade individuals to implement expected behaviors. In recent years, with the variety of electronic products and home appliances, the types and usage of batteries are constantly increasing, and the number of corresponding waste batteries is increasing. Because some of the waste batteries contain many heavy metals and electrolyte solutions, which will cause harm to the human body and the ecological environment, more and more people are paying attention to the pollution of waste batteries and their disposal. It has been demonstrated that visual prompts have the effects of protecting the environment. This paper attempts to study the effects of visual prompts on the recycling behavior of waste batteries and verify whether visual prompt has a positive effect on the recycling behavior of waste batteries by using a control group and an experimental group to compare the experimental methods. The visual prompts were pasted on the waste battery recycling bin and were carried out by GraphPad Prism in order to find an economic and effective way to promote people's recycling behaviors.
\end{abstract}

\section{Keywords}

Waste Batteries Recycling Behaviors, Visual Prompt, Effectiveness

\section{Introduction}

With the rapid development of the economy, various types of electrical appliances are increasing, and people's demand for batteries is increasing, and the recycling and disposal problems of used batteries are becoming more and more prominent. Usually, we will throw the used batteries away together with ordinary household waste. However, for batteries such as button batteries, recharge- 
able batteries, and automobile waste batteries, they mainly contain heavy metals such as cadmium and lead, and they also contain trace amounts of mercury. When they are abandoned in nature, mercury will slowly overflow from the battery, enter the soil or water source, and then enter the human body through crops, and damage the human kidney and nervous system. The exudation of cadmium and lead in the battery will also cause soil and water pollution, and also eventually enter the human body to create bodily damage in the liver and kidney and cause bone deformation in heavy cases. The data show that 3000 tons of used batteries can recover 141 tons of miscellaneous zinc ingots, 181 tons of electrolytic zinc, 260 tons of iron sheets, 300 tons of metallurgical manganese dioxide, 340 tons of electrolytic manganese dioxide, 340 tons of electrolytic manganese dioxide and 500 tons of iron sheet. The value is equivalent to the cost of developing two medium-sized mines in China. Therefore, after using the battery, we collect and recycle the used batteries in a unified manner to recycle resources, which can effectively protect the environment and promote green ecological development (Sussman, Greeno, Gifford, \& Scannell, 2013).

Studies have shown that $83 \%$ of people's information about their exposure to the outside world comes from vision. Visual prompt teaching refers to the use of visual prompt such as photos, text, line drawings, objects or videotapes to bring social expectations, social reviews and social tips (Wetzels, Boorsboom, van der Maas, \& Kievit, 2012). Visual prompts were firstly used in intervention strategies for autistic patients, with regarding to light-off behavior (Sussman \& Gifford, 2012), and energy conservation (Bergquist \& Nilsson, 2016) and studies in household food waste separation (Bernstad, 2014) have demonstrated that visual prompt can promote environmental behavior effectively. The visual prompts in this paper refer to the use of pictures, texts and other carriers for behavioral intervention, guiding and prompting individuals to make expected behaviors. Based on this, this paper attempts to study the effect of visual prompt on the behavior of recycling waste batteries, in order to provide a new perspective to effectively the behavior of recycling waste batteries.

\section{Methods}

\subsection{Research Object and Environment}

Two basically same residential areas in Nanjing, a city in China, were selected in this research, which area A was experimental group and area B was control group, and there were about 4000 residents live in these areas. Same waste batteries recycling bins were also set in these two areas respectively. In addition, we selected 4 volunteers as the recorders of the research experiment, expecting another two more as a supplement to other volunteers to a prevented unexpected situation. Two volunteers were responsible for counting the waste batteries recycling bins around 9:30 pm every day during the experiment, and centralized the waste batteries in the recycling bins to the waste recycling center. The others were responsible for recording and counting changes in the amount of waste batteries recovered. 


\subsection{Design for Visual Prompt}

As shown in Figure 1, our visual prompt poster was based on the advice of teachers, classmates and residents, as well as the reference to previous poster designs (Clayton \& Nesnidol, 2017). The visual prompt poster was mainly composed of pictures and texts. The picture was located at the top of the visual cue. It was a green recyclable sign. There were a green battery and three small people in the sign, indicating that the waste batteries can be recycled through recycling and protect the ecological environment effectively. The picture design was simple, and the theme of the visual cue was accurately conveyed through a simple logo. In addition, the design of the picture on the top of the poster can also attract people's attention more quickly and improve the visual prompt effect (Lin, Wang, Li, Gordon, \& Harder, 2016). In terms of text, the title "Please recycle waste batteries" under the picture is designed as large green bold text. The main content is that "A button cell can contaminate 600 tons of water, which is equivalent to the amount of water used by a person during his lifetime; one battery can contaminate 1 square meter of soil, making it permanently lost. Please recycle the hazardous waste batteries in your hands and take care of our eco-homes.". Through relevant research data, the hazards of discarding used batteries were pointed out, and the recycling of waste batteries was conducive to protecting the environment and deepening people's understanding of recycling waste batteries. The bottom yellow letter "The waste batteries placed in the recycling bin will be sent to the waste recycling center for centralized treatment." so that everyone knew the waste batteries in the recycling bins can be recycled effectively and increasing people's enthusiasm for recycling used batteries (Feng \& Zhang, 2019).

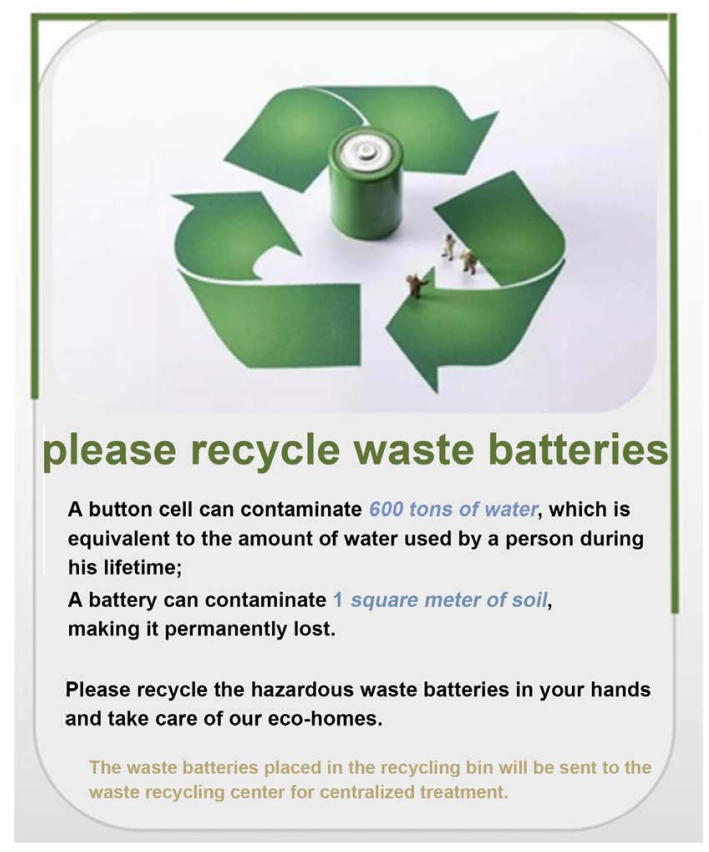

Figure 1. Visual prompt poster. 


\subsection{Procedures}

In this 23-week research, our purpose was to clarify the effect of visual poster for prompt people of recycling batteries. We compared the amount of recycled waste batteries in the recycling bin with or without visual poster in these two areas. For the bins in area B, there wasn't any treatment for them during the whole experiment. During the first 5 weeks, there was also nothing done for the bins in area $\mathrm{A}$, which were used to observe and compare the amount of recycled waste batteries in these two areas without visual prompt poster. During the following 5 weeks, the visual prompt poster designed by us was pasted on the recycling bins in area $\mathrm{A}$. The volunteers counted the changes in the number of waste batteries in these two areas during these weeks to observe the effectiveness of visual promote people' waste batteries recycling behavior. Finally, the visual prompt was continuously retained for 8 weeks, after that five-week follow-up to see if the visual prompt could have a sustained effect on promoting individuals' waste batteries recycling behaviors (van Meurs \& Aristoff, 2009).

\section{Results}

We got the mean and variance of the amount of waste batteries recycled in two communities per day during the three phases: baseline, the first 5 weeks, experimental, the following 5 weeks, and follow-up periods, the last 5 weeks, by two hypothesis tests; and then, the $P$ value, which meant the probability of an event, was calculated by GraphPad Prism statistical software. When $P>0.05$, there was no significant difference in the mean value of the data between the control group, area $\mathrm{B}$, and the experimental group, area $\mathrm{A}$. When $P<0.05$, the difference was significant in the mean value of the data between the control group and the experimental group, which meant that there was a significant difference in the amount of waste batteries recovered.

Table 1 showed the amount of waste batteries recovered in the experimental group and the control group during the baseline period. By calculating $P=$ $0.529>0.05$, it indicated that there was no significant difference in the amount of waste batteries recovered in the experimental group and the control group during the baseline period. Table 2 showed the amount of waste batteries recovered in the experimental group and the control group during the experiment, which is calculated by $P=0.016<0.05$, which meant that during the experiment, the amount of waste batteries recovered in the experimental group and the control group were significantly different, and the visual prompt poster of our design had a significant effect on promoting the individual's waste batteries recycling behavior. Finally, Table 3 showed the change in the amount of waste batteries recovery during the experimental period and the follow-up period. The statistics showed that $P=0.710>0.05$, which proved that the individuals' waste batteries recycling behaviors persisted during the follow-up period. The visual prompt was an effective way of promoting individuals' waste batteries recycling behaviors. 
Table 1. Number of waste batteries recovered in baseline period.

\begin{tabular}{ccc}
\hline Group & Mean $\pm \mathrm{SD}$ & $P$ \\
\hline Control group & $39.09 \pm 7.82$ & 0.529 \\
Treatment group & $41.45 \pm 9.41$ & \\
\hline
\end{tabular}

Table 2. Number of waste batteries recovered in experimental period.

\begin{tabular}{ccc}
\hline Group & Mean $\pm \mathrm{SD}$ & $P$ \\
\hline Control group & $40.55 \pm 10.58$ & 0.016 \\
Treatment group & $52.82 \pm 11.40$ & \\
\hline
\end{tabular}

Table 3. Number of waste batteries recovered of experimental group during experimental and follow-up period.

\begin{tabular}{ccc}
\hline Period & Mean \pm SD & $P$ \\
\hline Experimental period & $52.82 \pm 11.40$ & 0.710 \\
Follow-up period & $51.27 \pm 7.42$ & \\
\hline
\end{tabular}

\section{Discussion}

There is no doubt that visual prompts have a positive impact on the individuals' waste batteries recycling behaviors, and compare to no visual prompt, visual prompt can consistently promote the individuals' waste batteries recycling behaviors. There are several possible explanations for the impact of this visual prompt: First of all, from the way of obtaining information, the visual sensory contact is more humane than the simple object (waste battery recycling bin) and text. Secondly, the visual prompt poster has bright colors, vivid shape and strong sense of picture, which gives a good affinity to the senses. It has the characteristics of directness, extensiveness and interactivity which can enable people to consciously recycle waste batteries after seeing the visual prompt poster (Lee, Kurisu, \& Hanaki, 2013). Finally, the visual prompt poster is directly pasted on the waste battery recycling bin, after reading the visual cue, people can directly put the waste battery into the box, which greatly stimulates people's desire to recycle waste batteries.

Although the results of this study are in line with expectations, there are still some shortcomings. Firstly, our research object is two community residents, a total of about 8000 households. The number of research population is large and has a long follow-up time, so compliance is not easy to do well, and it maybe affects the evaluation of experimental effects. Secondly, because the visual prompt in this test is only attached to the waste battery recycling bin, due to the scope of the intervention measures, the selected research objects are not representative enough, and may affect the experimental results to some extent (Elson \& Przybylski, 2017). Finally, regarding the design of visual prompt poster, although many teachers and classmates have been consulted, there are still many shortcomings in visual senses. How to visually give people more impact and deepen 
people's enthusiasm for recycling behavior and Persistence makes visual cues more scientific? It is still waiting for later research.

\section{Conclusion}

This paper explored the influence of visual prompts on the waste batteries recycling behaviors. Through experimental research, the results showed that visual prompts had a significant impact on promoting the individuals' waste batteries recycling behaviors. In terms of the persistence of visual prompts, it can be seen from the observation of follow-up period that the individual recovery behavior persisted in the subsequent stage. In addition, due to the low cost of the visual prompt posters, the production process is simple; the picture is vivid and humorous; the content can be adjusted as needed, and it can be extended to other fields, such as "please donate your old clothes", "please sort and recycle garbage" and so on. In summary, it is an economical and effective way to promote individuals' waste batteries recycling behaviors and other environmentally friendly behaviors through visual prompts.

\section{Conflicts of Interest}

The authors declare no conflicts of interest regarding the publication of this paper.

\section{References}

Bergquist, M., \& Nilsson, A. (2016). I Saw the Sign: Promoting Energy Conservation via Normative Prompts. Journal of Environmental Psychology, 46, 23-31. https://doi.org/10.1016/j.jenvp.2016.03.005

Bernstad, A. (2014). Household Food Waste Separation Behavior and the Importance of Convenience. Waste Management, 34, 1317-1323. https://doi.org/10.1016/j.wasman.2014.03.013

Clayton, M., \& Nesnidol, S. (2017). Reducing Electricity Use on Campus: The Use of Prompts, Feedback, and Goal Setting to Decrease Excessive Classroom Lighting. Journal of Organizational Behavior Management, 37, 196-206. https://doi.org/10.1080/01608061.2017.1325823

Elson, M., \& Przybylski, A. K. (2017). The Science of Technology and Human Behavior: Standards, Old and New. Journal of Media Psychology, 29, 1-7. https://doi.org/10.1027/1864-1105/a000212

Feng, Z. L., \& Zhang, F. Z. (2019). Can Light-Shaped Visual Prompt Promote Individuals' Lights off Behaviors More Effectively than Ordinary One? Psychology, 10, 79-87. https://doi.org/10.4236/psych.2019.101006

Lee, H., Kurisu, K., \& Hanaki, K. (2013). Influential Factors on Pro-Environmental Behaviors-A Case Study in Tokyo and Seoul. Low Carbon Economy, 4, 104-116. https://doi.org/10.4236/lce.2013.43011

Lin, Z. Y., Wang, X., Li, C. J., Gordon, M. P. R., \& Harder, M. K. (2016). Visual Prompts or Volunteer Models: An Experiment in Recycling. Sustainability, 8, 458. https://doi.org/10.3390/su8050458

Sussman, R., \& Gifford, R. (2012). Please Turn off the Lights: The Effectiveness of Visual Prompts. Applied Ergonomics, 43, 596-603. 
https://doi.org/10.1016/j.apergo.2011.09.008

Sussman, R., Greeno, M., Gifford, R., \& Scannell, L. (2013). The Effectiveness of Models and Prompts on Waste Diversion: A Field Experiment on Composting by Cafeteria $\mathrm{Pa}$ trons. Journal of Applied Social Psychology, 43, 24-34.

https://doi.org/10.1111/j.1559-1816.2012.00978.x

van Meurs, L., \& Aristoff, M. (2009). Split-Second Recognition: What Makes Outdoor Advertising Work? Journal of Advertising Research, 49, 82-92.

https://doi.org/10.2501/S0021849909090011

Wetzels, R., Boorsboom, D., van der Maas, H. L. J., \& Kievit, R. A. (2012). An Agenda for Purely Confirmatory Research. Perspectives on Psychological Science, 7, 632-638.

https://doi.org/10.1177/1745691612463078 